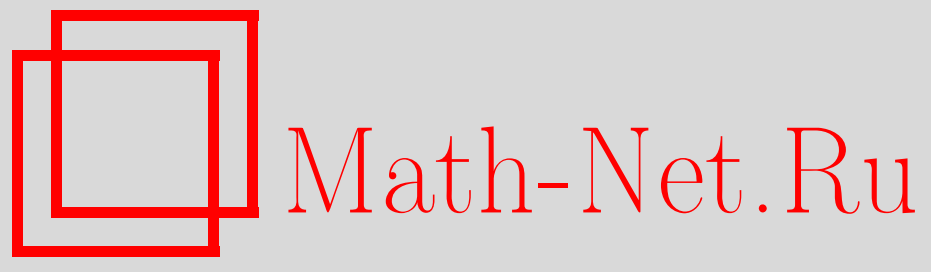

Х. Д. Икрамов, Об условиях совпадения сингулярных чисел $(3 \times 3)$-матриц с одноточечным спектром, Матем. заметки, 2006, том 80, выпуск 2, 187-192

DOI: https://doi.org/10.4213/mzm2798

Использование Общероссийского математического портала Math-Net.Ru подразумевает, что вы прочитали и согласны с пользовательским соглашением http://www.mathnet.ru/rus/agreement

Параметры загрузки:

IP : 54.237 .59 .107

26 апреля 2023 г., 13:20:31

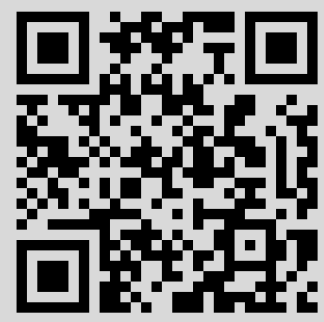




\section{ОБ УСЛОВИЯХ СОВПАДЕНИЯ СИНГУЛЯРНЫХ ЧИСЕЛ $(3 \times 3)$-МАТРИЦ С ОДНОТОЧЕЧНЫМ СПЕКТРОМ}

\section{Х. Д. Икрамов}

Для верхнетреугольной $(3 \times 3)$-матрицы $\Gamma_{a}$, все три диагональных элемента которой равны числу $a$, найдены необходимые и достаточные условия совпадения сингулярных чисел. Эти условия много проще равенства $\operatorname{discr} \varphi=0$, где в левой части стоит дискриминант характеристического многочлена $\varphi$ матрицы $G_{a}=\Gamma_{a}^{*} \Gamma_{a}$. Понимание характера поведения сингулярных чисел матрицы $\Gamma_{a}$ важно в задаче о нахождении матрицы с тройным собственным значением нуль, ближайшей к заданной нормальной матрице $A$.

Библиография: 2 названия.

1. Введение. Пусть $M_{n}(\mathbb{C})$ - множество комплексных $(n \times n)$-матриц, а $\mathscr{L}$ его подмножество, состоящее из матриц с кратным собственным значением нуль. Для произвольной матрицы $A \in M_{n}(\mathbb{C})$ определим (спектральное) расстояние до множества $\mathscr{L}$ формулой

$$
\rho_{2}(A, \mathscr{L})=\min _{L \in \mathscr{L}}\|A-L\|_{2}
$$

В [1] показано, что

$$
\rho_{2}(A, \mathscr{L})=\max _{\gamma \geqslant 0} \sigma_{2 n-1}(F(\gamma))
$$

где

$$
F(\gamma)=\left(\begin{array}{cc}
A & \gamma I_{n} \\
0 & A
\end{array}\right)
$$

a $\sigma_{2 n-1}(F(\gamma))$ - предпоследнее сингулярное число матрицы $F(\gamma)$ при упорядочении сингулярных чисел по убыванию.

Для нормальной матрицы $A \in M_{n}(\mathbb{C})$ с собственными значениями $\lambda_{1}, \ldots, \lambda_{n}$, где

$$
\left|\lambda_{1}\right| \geqslant\left|\lambda_{2}\right| \geqslant \cdots \geqslant\left|\lambda_{n}\right|,
$$

в [2] найдена формула

$$
\rho_{2}(A, \mathscr{L})=\left(\frac{\left|\lambda_{n-1}\right|^{2}+\left|\lambda_{n}\right|^{2}}{2}\right)^{1 / 2} .
$$

(C) Х. Д. Икрамов, 2006 
Вывод этой формулы основывался на равенстве (1) и исследовании поведения сингулярных чисел матриц вида

$$
\left(\begin{array}{ll}
a & \gamma \\
0 & a
\end{array}\right)
$$

При фиксированном $a \neq 0$ старшее сингулярное число такой матрицы есть монотонно возрастающая и неограниченная функция неотрицательного параметра $\gamma$, а младшее сингулярное число монотонно убывает к нулю.

Рассмотрим теперь множество $\mathscr{M} \subset \mathscr{L}$, состоящее из $(n \times n)$-матриц с собственным значением нуль кратности по меньшей мере три (таким образом, $n \geqslant 3$ ). Пусть, как и прежде, $A$ - нормальная $(n \times n)$-матрица. Определение расстояния $\rho_{2}(A, \mathscr{M})$ требует исследования поведения сингулярных чисел матриц вида

$$
\Gamma_{a}(\gamma)=\left(\begin{array}{ccc}
a & \gamma_{1} & \gamma_{3} \\
0 & a & \gamma_{2} \\
0 & 0 & a
\end{array}\right)
$$

как функций от трехмерного векторного параметра

$$
\gamma=\left(\gamma_{1}, \gamma_{2}, \gamma_{3}\right)
$$

Подобное исследование оказывается значительно более сложным, чем для двумерных матриц (2). В частности, нетривиальным оказывается вопрос об условиях совпадения сингулярных чисел матрицы $\Gamma_{a}(\gamma)$.

Заметим, что подлинной особенностью матрицы (3) является не треугольность ее формы (согласно теореме Шура к треугольному виду посредством унитарного подобия можно привести всякую комплексную квадратную матрицу), а одноточечность ее спектра. Если $a \neq 0$, то, пользуясь равенством $\Gamma_{a}(\gamma)=a \Gamma_{1}(\gamma / a)$, можно без ограничения общности считать, что $a=1$. Далее, выполняя с матрицей (3) подходящее диагональное унитарное подобие, можно заменить комплексные числа $\gamma_{1}$ и $\gamma_{2}$ их модулями. Поэтому в дальнейшем мы считаем $\gamma_{1}$ и $\gamma_{2}$ вещественными неотрицательными числами.

Имеется очевидный подход к описанию условий совпадения сингулярных чисел матрицы (3). Квадраты этих чисел суть собственные значения матрицы $G_{a}=\Gamma_{a}^{*} \Gamma_{a}$, иначе говоря, корни ее характеристического многочлена

$$
\lambda^{3}+\alpha_{1} \lambda^{2}+\alpha_{2} \lambda+\alpha_{3}
$$

где

$$
\begin{gathered}
\alpha_{1}=-\operatorname{tr} G_{a}=-\left(3|a|^{2}+\gamma_{1}^{2}+\gamma_{2}^{2}+\left|\gamma_{3}\right|^{2}\right), \\
\alpha_{2}=3|a|^{4}+\left(\gamma_{1}^{2}+\gamma_{2}^{2}+\left|\gamma_{3}\right|^{2}\right)|a|^{2}+\gamma_{1}^{2} \gamma_{2}^{2}-2 \gamma_{1} \gamma_{2} \operatorname{Re}\left(\gamma_{3} a\right), \\
\alpha_{3}=-\left|\operatorname{det} \Gamma_{a}\right|^{2}=-|a|^{6} .
\end{gathered}
$$

Многочлен (5) имеет кратный корень тогда и только тогда, когда равен нулю его дискриминант

$$
D=\alpha_{1}^{2} \alpha_{2}^{2}-4 \alpha_{2}^{3}-4 \alpha_{1}^{3} \alpha_{3}+18 \alpha_{1} \alpha_{2} \alpha_{3}-27 \alpha_{3}^{2} .
$$


Будем в соответствии со сказанным выше считать, что $a=1$, а $\gamma_{1}$ и $\gamma_{2}$ вещественны и неотрицательны. Для комплексного (в общем случае) параметра $\gamma_{3}$ примем представление $\gamma_{3}=\xi+i \eta$. Подстановка в (6) формул для коэффициентов $\alpha_{1}, \alpha_{2}$ и $\alpha_{3}$ приводит к громоздкому выражению, содержащему более 120 мономов от $\gamma_{1}, \gamma_{2}, \xi$ и $\eta$.

Тем контрастнее выглядит следующий результат, доказываемый в п. 2 настоящей статьи.

Теорема 1. Пусть $\gamma_{1}, \gamma_{2} \in \mathbb{R}^{+}, a \gamma_{3}=\xi+i \eta$. Если $a=1$, то необходимым и достаточным условием совпадения сингулярных чисел матрицы $\Gamma_{1}(\gamma)$ является выполнение равенств

$$
\gamma_{1}=\gamma_{2} \equiv \gamma_{0}, \quad \gamma_{0}^{2}=\frac{\xi^{2}}{1+\xi}, \quad \eta=0 .
$$

Соответствующее кратное сингулярное число равно

$$
\sigma=\frac{1}{\sqrt{1+\xi}}
$$

При $а=0$ для совпадения сингулярных чисел необходимо и достаточно, чтобъ

$$
\gamma_{1} \gamma_{2}=0 \quad \text { либо } \quad \gamma_{1}=\gamma_{2} \equiv \gamma_{0}, \quad \gamma_{3}=0 .
$$

В первом случае кратным сингулярным числом матрицы $\Gamma_{0}(\gamma)$ является нуль; во втором случае сингулярные числа равны $\gamma_{0}, \gamma_{0}, 0$.

2. Доказательство теоремы. Доказательство теоремы начнем с более простого случая $a=0$. Очевидно, что одним из сингулярных чисел при любых $\gamma_{1}, \gamma_{2}, \gamma_{3}$ является $\sigma=0$. Два других числа суть одновременно сингулярные числа $(2 \times 2)$ матрицы

$$
\left(\begin{array}{cc}
\gamma_{1} & \gamma_{3} \\
0 & \gamma_{2}
\end{array}\right)
$$

Квадраты этих чисел являются корнями квадратного уравнения

$$
x^{2}-\left(\gamma_{1}^{2}+\gamma_{2}^{2}+\left|\gamma_{3}\right|^{2}\right) x+\left(\gamma_{1} \gamma_{2}\right)^{2}=0 .
$$

При $\gamma_{1} \gamma_{2}=0$ один из корней равен нулю и $\sigma=0$ есть кратное сингулярное число матрицы $\Gamma_{0}(\gamma)$. Если же $\gamma_{1} \gamma_{2} \neq 0$, то дискриминант уравнения (10) имеет вид

$$
d=\left(\gamma_{1}^{2}+\gamma_{2}^{2}+\left|\gamma_{3}\right|^{2}\right)^{2}-4\left(\gamma_{1} \gamma_{2}\right)^{2}=\left\{\left(\gamma_{1}-\gamma_{2}\right)^{2}+\left|\gamma_{3}\right|^{2}\right\}\left\{\left(\gamma_{1}+\gamma_{2}\right)^{2}+\left|\gamma_{3}\right|^{2}\right\}
$$

В этом случае условиями совпадения двух ненулевых сингулярных чисел матрицы $\Gamma_{0}(\gamma)$ являются равенства $(9)$.

Перейдем к случаю $a=1$. Кратное сингулярное число $\sigma$ матрицы $\Gamma_{1}(\gamma)$, будучи возведено в квадрат, дает кратное собственное значение $\lambda=\sigma^{2}$ матриц

$$
\begin{aligned}
& G_{1}(\gamma)=\Gamma_{1}^{*}(\gamma) \Gamma_{1}(\gamma)=\left(\begin{array}{ccc}
1 & \gamma_{1} & \gamma_{3} \\
\gamma_{1} & 1+\gamma_{1}^{2} & \gamma_{1} \gamma_{3}+\gamma_{2} \\
\bar{\gamma}_{3} & \gamma_{1} \bar{\gamma}_{3}+\gamma_{2} & 1+\gamma_{2}^{2}+\left|\gamma_{3}\right|^{2}
\end{array}\right) \\
& F_{1}(\gamma)=\Gamma_{1}(\gamma) \Gamma_{1}^{*}(\gamma)=\left(\begin{array}{ccc}
1+\gamma_{1}^{2}+\left|\gamma_{3}\right|^{2} & \gamma_{1}+\gamma_{2} \gamma_{3} & \gamma_{3} \\
\gamma_{1}+\gamma_{2} \bar{\gamma}_{3} & 1+\gamma_{2}^{2} & \gamma_{2} \\
\bar{\gamma}_{3} & \gamma_{2} & 1
\end{array}\right)
\end{aligned}
$$


Поскольку матрицы (11) и (12) эрмитовы, кратное собственное значение $\lambda$ является собственным значением и для каждой их главной $(2 \times 2)$-подматрицы. В частности, $\lambda$ есть собственное значение $(2 \times 2)$-матриц

$$
\left(\begin{array}{cc}
1 & \gamma_{1} \\
\gamma_{1} & 1+\gamma_{1}^{2}
\end{array}\right), \quad\left(\begin{array}{cc}
1+\gamma_{2}^{2} & \gamma_{2} \\
\gamma_{2} & 1
\end{array}\right) .
$$

Собственные значения матрицы

$$
\left(\begin{array}{cc}
1 & \gamma_{1} \\
\gamma_{1} & 1+\gamma_{1}^{2}
\end{array}\right)
$$

равны

$$
\nu_{1,2}=1+\frac{\gamma_{1}^{2}}{2} \pm\left(\gamma_{1}^{2}+\frac{\gamma_{1}^{4}}{4}\right)^{1 / 2} .
$$

Они совпадают при $\gamma_{1}=0$. При $\gamma_{1} \neq 0$ больший (соответственно меньший) из корней строго монотонно растет (соответственно убывает) как функция от $\gamma_{1}^{2}$. Аналогичные заключения (с заменой $\gamma_{1}$ на $\gamma_{2}$ ) справедливы для матрицы

$$
\left(\begin{array}{cc}
1+\gamma_{2}^{2} & \gamma_{2} \\
\gamma_{2} & 1
\end{array}\right)
$$

(сводящейся к матрице вида (14) посредством транспозиции строк и столбцов).

Итак, $\lambda$ тогда и только тогда может быть собственным значением сразу для обеих матриц в (13), когда

$$
\gamma_{1}=\gamma_{2}
$$

В дальнейшем анализе мы считаем это условие выполненным и полагаем $\gamma_{0} \equiv$ $\gamma_{1}=\gamma_{2}$. Поэтому характеристическое уравнение матрицы (14) можно записать как

$$
x^{2}-\left(2+\gamma_{0}^{2}\right) x+1=0 .
$$

Обратимся теперь к главной подматрице матрицы $G_{1}(\gamma)$, расположенной в строках и столбцах с номерами 1 и 3 . Ее характеристическое уравнение:

$$
x^{2}-\left(2+\gamma_{0}^{2}+\left|\gamma_{3}\right|^{2}\right) x+\left(1+\gamma_{0}^{2}\right)=0 .
$$

Будучи корнем уравнений (15) и (16), $\lambda$ является корнем и для их разности, т.е. для уравнения

$$
-\left|\gamma_{3}\right|^{2} x+\gamma_{0}^{2}=0
$$

При $\gamma_{3}=0$ отсюда следует, что $\gamma_{0}=0$ и все условия (7), а также равенство (8) выполнены. Если $\gamma_{3} \neq 0$, то из (17) находим, что

$$
\lambda=\frac{\gamma_{0}^{2}}{\left|\gamma_{3}\right|^{2}}
$$

В матрице $G_{1}(\gamma)$ все еще не рассмотрена главная подматрица

$$
\left(\begin{array}{cc}
1+\gamma_{0}^{2} & \gamma_{0}\left(1+\gamma_{3}\right) \\
\gamma_{0}\left(1+\bar{\gamma}_{3}\right) & 1+\gamma_{0}^{2}+\left|\gamma_{3}\right|^{2}
\end{array}\right),
$$


расположенная в строках и столбцах с номерами 2 и 3. Комбинируя ее характеристическое уравнение

$$
x^{2}-\left(2+2 \gamma_{0}^{2}+\left|\gamma_{3}\right|^{2}\right) x+\left(1+\gamma_{0}^{2}+\gamma_{0}^{4}+\left|\gamma_{3}\right|^{2}-2 \gamma_{0}^{2} \xi\right)=0
$$

с уравнением (16), получим линейное уравнение

$$
-\gamma_{0}^{2} x+\left(\gamma_{0}^{4}+\left|\gamma_{3}\right|^{2}-2 \gamma_{0}^{2} \xi\right)=0
$$

При $\gamma_{0}=0$ отсюда следует, что $\gamma_{3}=0$. Этот случай был рассмотрен выше. Если же $\gamma_{0} \neq 0$, то число (18) должно быть решением уравнения (20), откуда получаем соотношение

$$
-\gamma_{0}^{4}+\gamma_{0}^{4}\left|\gamma_{3}\right|^{2}+\left|\gamma_{3}\right|^{4}-2 \gamma_{0}^{2}\left|\gamma_{3}\right|^{2} \xi=0
$$

До сих пор использовались лишь следствия характеристических уравнений (15), (16) и (20), но не сами эти уравнения. Запишем теперь тот факт, что число (18) является корнем уравнения (15), а потому и двух других уравнений:

$$
\gamma_{0}^{4}-\gamma_{0}^{4}\left|\gamma_{3}\right|^{2}+\left|\gamma_{3}\right|^{4}-2 \gamma_{0}^{2}\left|\gamma_{3}\right|^{2}=0
$$

Складывая (21) и (22), имеем

$$
\left|\gamma_{3}\right|^{4}=\gamma_{0}^{2}\left|\gamma_{3}\right|^{2}(1+\xi)
$$

откуда следует, что

$$
\xi>-1
$$

(при $\xi=-1$ получим уже рассмотренный случай $\gamma_{3}=0$ ) и

$$
\left|\gamma_{3}\right|^{2}=\gamma_{0}^{2}(1+\xi)
$$

Сопоставляя это равенство с (18), находим

$$
\lambda=\frac{1}{1+\xi},
$$

что эквивалентно соотношению (8).

Вычтем теперь $(21)$ из $(22)$ и сократим полученное равенство на $2 \gamma_{0}^{2}$. Это дает

$$
\gamma_{0}^{2}=\left|\gamma_{3}\right|^{2}\left(1-\xi+\gamma_{0}^{2}\right)
$$

Заменяя $\left|\gamma_{3}\right|^{2}$ в правой части в соответствии с (23) и сокращая на $\gamma_{0}^{2}$, приходим к соотношению

$$
1=(1+\xi)\left(1-\xi+\gamma_{0}^{2}\right)
$$

откуда

$$
\xi^{2}=\gamma_{0}^{2}(1+\xi)
$$

Сопоставляя это равенство с (23), заключаем, что

$$
\left|\gamma_{3}\right|^{2}=\xi^{2}
$$


т.е. $\eta=0$. Таким образом, показана необходимость всех условий (7) и справедливость формулы (8).

Достаточность условий (7) устанавливается путем прямой подстановки выражения (24) в левые части уравнений (5), (15), (17) и (20). Тот факт, что результат подстановки всякий раз оказывается равным нулю, означает, что $\lambda$ есть собственное значение самой матрицы $G_{1}(\gamma)$ и трех ее главных подматриц порядка 2. Иначе говоря, определитель и главные миноры порядка 2 эрмитовой матрицы $G_{1}(\gamma)-\lambda I_{3}$ равны нулю, откуда следует, что ее ранг не превосходит единицы, т.е. $\lambda$ есть кратное собственное значение матрицы $G_{1}(\gamma)$, а $\sigma=\sqrt{\lambda}$ есть кратное сингулярное число матрицы $\Gamma_{1}(\gamma)$. Теперь теорема доказана полностью.

\section{СПИСОК ЦИТИРОВАННОЙ ЛИТЕРАТУРЫ}

[1] A. N. Malyshev, "A formula for the 2-norm distance from a matrix to the set of matrices with multiple eigenvalues", Numer. Math., 83 (1999), 443-454.

[2] Х. Д. Икрамов, А. М. Назари, "Об одном замечательном следствии формулы Малышева", Докл. РАН, 385:5 (2002), 599-600.

\section{Х. Д. Икрамов}

Поступило

Московский государственный университет

26.04.2005

им. М. В. Ломоносова

Исправленный вариант

E-mail: ikramov@cs.msu.su

19.12.2005 